\title{
Identifying and Examining Densely Populated Areas with the Influence of Weather Change on Transport
}

\author{
M. Siva Shankar, J. Saritha
}

\begin{abstract}
In this work we identifying and examining the weather conditions on metropolitan area by using Big data. In this data describe the collection of data very large in size. In some urban regions transporting problem will be arrived in the changing on weather. Now this work understands the various characteristics overall city in urban areas. Some of the urban areas major pollution problem that can arrive in the motor vehicles / industries from metrological data. The data compilation process will be available in government web sites. This website used for making of see and retrieving the previous data and compare with the real data. To estimate the data mining by using Collaborative Filtering algorithm. Now going to this algorithm to estimate the real data and compare next day data.
\end{abstract}

Key Terms: Collaborative Filtering Algorithm, weather forecast, Track analysis, MVC, Web service

\section{INTRODUCTION}

In urban areas population will be more. The people's live in continuous improvement on the urban areas. The environment will be pollutant in many situations by using of vehicles. In villages are green in area there is no pollution because of peoples they develop agriculture in own land. The urban areas peoples can have the own land but the land will be provided to construct the buildings and roads. There are several categories in urban area they are transportation, urban planning, environment energy, public safety, energy, and security. So that time too many problems also arrived. Now the aim to solve the cities problems such as traffic control, energy saving, and pollution based on the flow of the traffic data and geographical data [1] In various cities investment for influence of traffic in weather changing. One of the example in rain season heavy rain fall the problem to slow down traffic and in the rain season cause congestions due to visibility will be low and demand of vehicle will high. The temperature will decrease in the cold days.

We Identifying the weather changes in summer season, winter season and raining season. by identifying the traffic variations on the weather conditions by using weather-traffic index. Weather-traffic index formations mean to collect the data from entire city. Shanghai is the largest city. In this city traffic will be more and more number of vehicles also there, the formation of data will be collect the shanghai is $115.2 \mathrm{~GB}$

Revised Version Manuscript Received on 16 September, 2019.

M. Siva Shankar, M. Tech Student, Dept Of Computer Science And Engineering, Jntua College Of Engineering, Pulivendula, Andhra Pradesh, India (Email: sivasiva4316@ gmail.com)

Dr J. Saritha, Assistant Professor, Department Of Computer Science And Engineering, Jntua College Of Engineering, Pulivendula-516390, Andhra Pradesh, India for the fast 4 years [2].

The road network also being complicated in weather changes. now the roads will be extracted in changing weather conditions, at that time traffic controlling very complicated. So provide the data for identifying the pollution level at entire city environment. By using the collaborative filtering algorithm identifying the previous data compare with next day data through web sites. In this data will providing the actual data and predict data.

\section{RELATED WORK}

\subsection{Big data:}

Big data is the collection data huge in size, to analyze the extract information from the data sets, the data will be formed in three types, structured data, unstructured data and semi structured data. There are three characteristics they are: volume, variety and velocity. volume is the quantity of generated and stored data. Variety is the type and nature of the data; people analyze the using the result. Velocity

is the speed at which data at which processing and generated. The technologies providing the big data is databases, data mining, distributed file systems etc.

\subsection{Data Mining:}

Data mining is used for solving the problems, mining the knowledge data from large data sets and also identify the patterns. It is also information processing system like collection, extraction, warehousing. The knowledge discovery databases (KDD) process is different stages: they are selection, pre-processing, transformation, data mining and interpretation. Different methods use to data mining cluster analysis, neural network, decision tree etc...

The urban population will be increased by around 2.7 billion people in between 2014 to 2050 . The population will be reached in around 70\%.the economic potential raised on products [3]. The traffic problem arrived in the city. Pollution levels also increase the urban areas. City problems such as energy consumption, pollution based on the traffic flow and geographical data. The weather-traffic index system may be providing the entire city formation data will be providing and also providing the key factor analysis.

In the urban area of Beijing is the network between the pollution and population. The functional urban area of Beijing is mining the taxies GPS trajectories. The taxies may 
be providing the pollution, the pollution level largely modulated by metrological conditions. In the Beijing health problem will be arrived topublic [4]. So providing the ventilate and clean atmosphere in the pollution transport. The communication and transporting problem will arrive in the urban area. To developing the urban computing and analysis tools in cities [5]. Mainly we have to focus on the pollution data, mining the all information about the weather changes in the city environment.

By using collaborative filtering algorithm identifying weather data. This data will compare with actual data to next day data through the web service. Now the next day data fine particulates change on the weather change on transport. In various seasons the road construction will be different at that time traffic level also changes. so everyday changes the weather conditions.

\section{PROPOSED WORK}

The climate will be change in urban areas, the transportation problem may have arrived in the system. In the several problems in the global sector, one of the problem is pollution [6]. In this proposed work aim detailed information about the pollution levels throughout city environment. Mining the previous data compare with the next day data through the web service. By using Big data to build a algorithm technique identifying the entire information around the pollution levels and compare with the real data and previous data through the government web site.

The Central Pollution Control Board web service will be providing the entire city environment. The pollution levels may compare with the real data and next day data. Model-view-controller technique will be used in the identifying the pollution levels and also examine the data.

\subsection{Advantages:}

$>$ Automation of prognosis model

$>$ More securable

$>$ It identifies pollution level day by day

$>$ It improves the performance of road network

$>$ It provides quick access to the weather conditions

$>$ Reliability is more

\subsection{System Architecture:}

The below figure shows the system architecture may consist the location server and main server. The location server is also consisting display system, user GUI, current environment data, web service. The main server also consists data sets, location details, excel API, data, mining technique and also administrator.

\subsubsection{User:}

The working of user giving and register the data, by getting the user name and password, user id etc. User store the information about in the registers, anyone can access the data giving the permission to user. The user account will be login. The User giving the data through location sever, he will register the environment data. Now he will store the location data current environment data main server. In the location server mainly consisting the web predictive model. Now presenting the collaborative filtering

The working as location server input between the user and

service, the web service to get extract input to the location server sending another web service in the main server The main server also storing some of the data. the getting output by using web service.

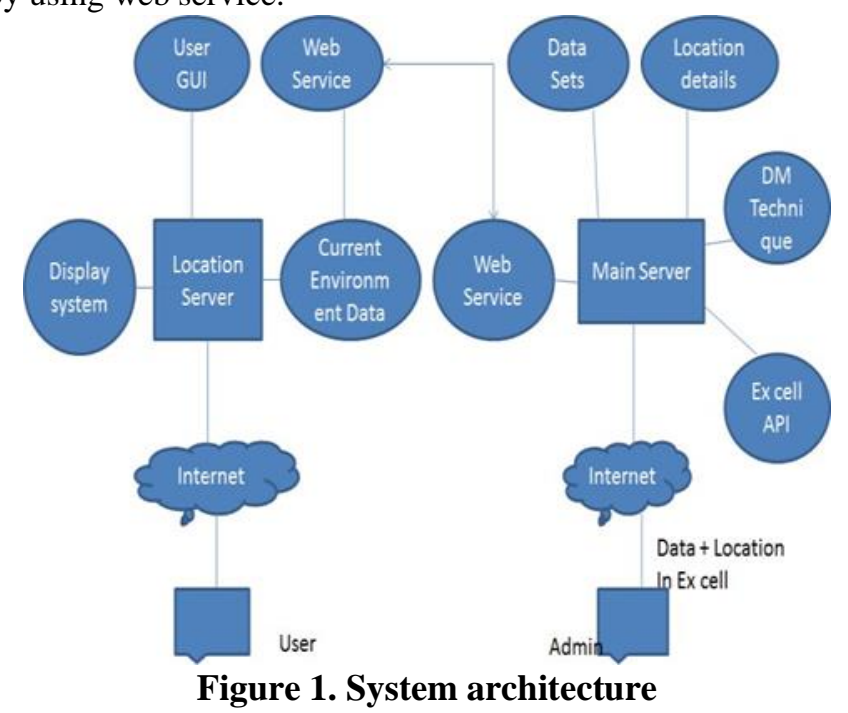

Figure 1 explains about the architecture of the proposed work. In this architecture we have:

\subsubsection{Admin:}

Admin may consist the main server. In the server one web service link with location server. The admin working as some changes about the main server such as add city details, upload the data and view city details. Admin permission to access only authorized persons. He got one account, login to this account by using admin name and password. The present data may be consisting date and time of the entire environment city, in the date and time data will be uploaded in the application. The

database server may also be storing the date and time of the data. The real time data set in different cities watch the one particular website.

http://www.cpcb.gov.in/CAAQM/mappage/f rmindiamap.aspx

\subsubsection{Forecast:}

In this forecast to get pollution levels by using collaborative filtering algorithm at particular location. Select a particular forecast location similarity with the all locations, to select a number of locations similarity with correlation persons and vector rating.

\subsection{Collaborative Filtering Algorithm:}

Collaborative filtering is one the technique commonly used to build personalized recommendations on the web. it is used to automatic prediction about a person, most popular companies like Amazon, Netflix and IMDB uses the collaborative filtering technology in the web sites. There are different types of collaborative filtering they are: (1)Memory based : it makes use of interact between the user and items. The user information rating will be calculated by using recommendations. (2)Model based : it will used for to come 
up to forecast for original data. models are created by using data mining. (3)Hybrid : the number of programs will combine the memory based and model based. The collaborative filtering algorithm is the compare with the previous data and next day data. Every day see the data updated to the local server and main server. The admin and user want to get the pollution details by the entire city environment. The data mining will be used to forecast the pollution levels every day.

\subsection{Web Service:}

It is a one of the service communication between the two electronic devices through world wide web. Some other technologies in the web services are HTTP, XML, JSON etc. the web API implements web services.

In this work the web service will be used to communication between the location server and main server. The web service provides the find out the overall pollution levels up to day date through the city environment. The web service may receive the user and admin requests through the network.

\subsection{Model-View-Controller (MVC):}

The model-view-controller is one of the technique used in the work. The model is responsible for managing the data application. It receives the output from the controller. The view model responsible for model format. The controller is used to interaction between the user input and model. Model-view-controller provide some of the programming languages. they are: Python, JSON etc.

In this work MVC will be used to managing previous data and next day data. To view the pollution levels environment in the entire city. The MVC makes the located the user side and admin side, the admin may use to MVC by receives the information for main server.

\section{RESULT AND ANALYSIS}

The urban areas population will be more and the transportation problem arrived in the traffic at that time pollution raised in the city environment. To identify the pollution data levels at actual data and compare with the next day data through the web service as Control Pollution Central Board. The pollution levels will be updated change on weather conditions up to date. To selecting one particular location on the city to check out the weather conditions and pollution levels.

Table 1. Comparing the pollution data

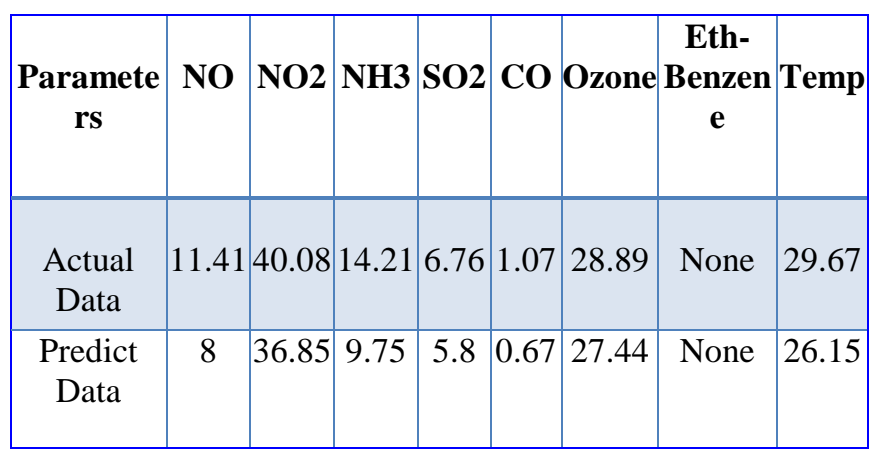

Table 1 shows the comparing the pollution data at present data and next day data, in that pollution different parameter are available, the parameters values may be different depending on changing weather conditions

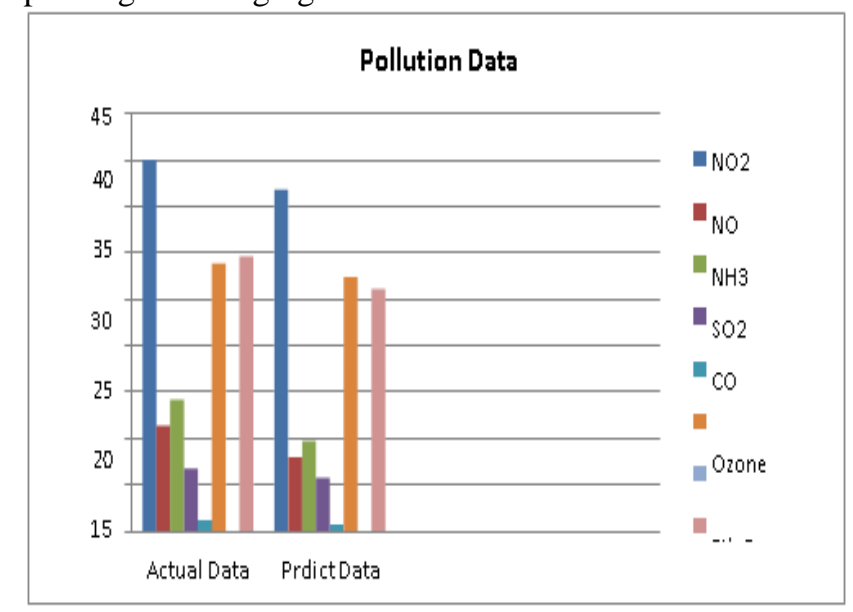

Figure 2. pollution data in different parameters

The figure 2 shows the pollution data levels at different parameters in the weather change on environment the city levels. The weather levels up to date changing on environment, the parameter are $\mathrm{NO}, \mathrm{NH} 3, \mathrm{SO} 2, \mathrm{NO} 2, \mathrm{CO}$, Ozone, Eth-

Benzene, Temp etc. So these parameter values may be changed in actual data and predict data.

\section{CONCLUSION AND FUTURE WORK}

It is mainly focus on the weather change on transport. The population will be growing on day by day. The pollution levels may be change on weather condition in the urban areas. Now we are implementing the collaborative filtering algorithm to identifying the pollution levels changing on weather conditions entire the city. In this work mining the previous data comparing the next day data. In future work, to controlling the pollution levels in urban areas and avoiding the transportation problems. To get control the traffic problems, save the trees and plants. Limited space to provide the organizations. At present system we are using collaborative filtering algorithm.

\section{REFERENCES}

1. O. Wolfson, L.Capra, Y.Zheng, H.Yang, 2014, Urban Computing: Concepts, Methodologies.

2. Mingxuan Yuan, Yanhua Li, Haoyu Tan, Fellow, Ye N Ding, Ke Deng, Lionel M. Ni Senior Member, IEEE, November- 2015, Dissecting Regional Reather Traffic Sensitivity throughout a City.

3. Highlights of The World Urbanization in the year 2014 Department of Economic and Social AffairS

4. K.Xu, Jichang Zhao, X.Liang, , Ruiwen Li 2015 , Segmentation and Evolution of urban areas in Beijing: a view from mobility data of massive individuals.

5. S. Wang, G.Forestier, C.F..Eick , A.Puissant, Z.Cao, C.F..Eick , 2013, Analyzing the Composition of Cities Using Spatial Clustering.

6. Piet. Rietveld and M. J. Koetse , IEEE, 2009, Impact of Climate Change and Weather On Transport: An Overview of Empirical Findings. 